\title{
Two-dimensional Modal Beamforming in Wavenumber Space for Duct Acoustics
}

\author{
Robert P. Dougherty ${ }^{1}$ \\ OptiNav Inc., Bellevue, Washington, 98005, USA \\ and \\ Richard F. Bozak ${ }^{2}$ \\ NASA Glenn Research Center, Cleveland, Ohio, 44135, USA
}

\begin{abstract}
As turbofan bypass ratio continues to increase, civilian aircraft noise is increasingly dominated by fan noise. Fan noise propagating from its rotor and stator origins to the community passes through the inlet or aft flow duct, where its confined situation makes it susceptible to characterization by wall-mounted microphone arrays. Recently, the NASAGlenn Research Center adapted its W-8 Single Stage Axial Compressor Facility to this type of measurement. OptiNav, Inc. took the opportunity to improve and simplify the duct mode processing in its Beamform Interactive computer program. A new approach to in-duct beamforming with a 2D wall-mounted array of microphones was developed. The purpose of this paper is to document the beamforming approach and provide some sample results from the $\mathbf{W - 8}$ facility.
\end{abstract}

\section{Nomenclature}

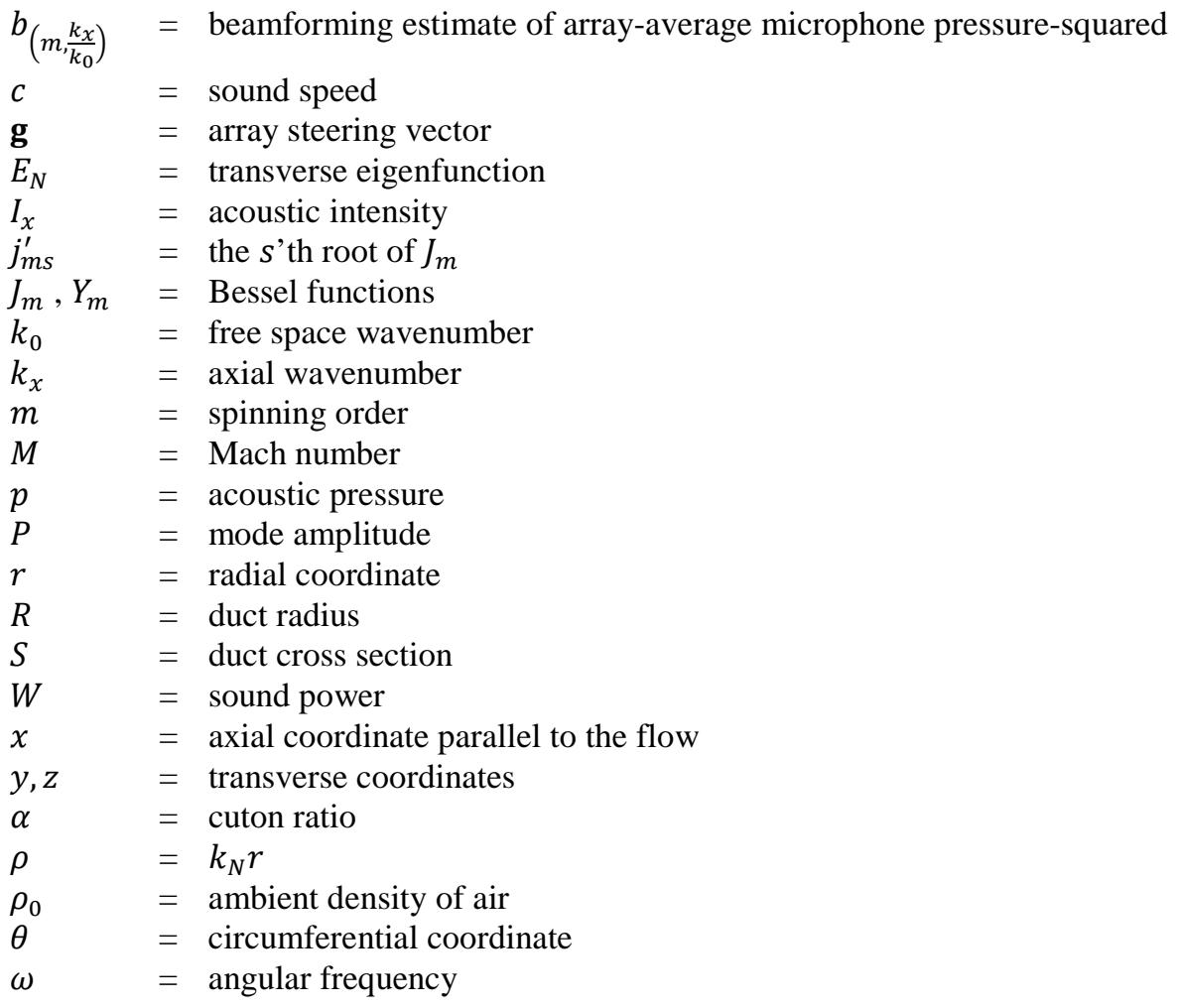

\footnotetext{
${ }^{1}$ President, AIAA Associate Fellow.

${ }^{2}$ Research Aerospace Engineer, Acoustics Branch, AIAA Member.
} 


\section{Introduction}

Fan noise is an increasingly important component of the community noise of transport aircraft. NASA recently applied its W-8 Single Stage Axial Compressor Facility[1] to investigate the effectiveness of several over-the-rotor treatment designs [2]. The treatments were compared on the basis of in-duct power level (PWL). OptiNav's post-processing beamforming code, Beamform Interactive, was revised to support the computation of PWL using data from an array of wall mounted pressure transducers in the inlet of $\mathrm{W}-8$. The purpose of this paper is to document the new beamforming method which was developed for this computation.

The method is designed for broadband noise. The PWL is computed from sum of the estimated powers in the outward propagating cuton modes. The mode powers are obtained by applying a beamforming technique to the microphone signals. This approach assumes that the modes are mutually incoherent [3]. As in previous work [4-7], the arrangement of microphones in the W-8 is a cross array combining an circumferential subarray and an axial subarray.

The beamforming used in Refs. [4-6] was based on computing steering vectors for individual modes. CLEAN-SC deconvolution [8] was applied in Ref. [4], DAMAS [9] and CLEAN-SC were used in Ref. [5], and Functional Beamforming [10] was applied in Ref. [6].

The new method uses a beamforming grid in the $m-\frac{k_{x}}{k_{0}}$ plane, where $m$ is the spinning order and $\frac{k_{x}}{k_{0}}$ is the axial wavenumber, normalized by the free space wavenumber. The beamforming values are estimates of the array-average pressure-squared values corresponding to each $m-\frac{k_{x}}{k_{0}}$ grid point. Distinct propagating modes, if present, appear as peaks in the beamform map, but it not necessary to know the modal values of $\frac{k_{x}}{k_{0}}$ in advance of the beamforming. This choice was made to simplify the beamforming and make it more robust by removing the dependence on details such as the Mach number and the temperature. It is more robust because errors in computing the theoretical modal values of $\frac{k_{x}}{k_{0}}$ no longer create errors in the steering vectors; they only shift the peaks in the beamform map. The beamform maps are post-processed by identifying peaks and then assigning them to the modes with the nearest theoretical $m$ $\frac{k_{x}}{k_{0}}$ values. Once the modal pressure-squared values have been determined, they can be converted into mode powers and summed. The sum is expressed in $\mathrm{dB}$ to give the PWL. It is also possible to use a modal radiation model to sum the modes in the far field to estimate the directivity of the fan if it were installed with an open inlet as opposed to the confined compressor facility.

A new beamforming algorithm, Quantitative Bemforming (QB), is applied to the $m-\frac{k_{x}}{k_{0}}$ grid. This method can be described as Functional Beamforming with $v=-1$, combined with a special regularization technique that removes Functional Beamforming's sensitivity to steering vector errors. In the present application, this regularization is not critical as it might be because the chosen grid lends itself to accurate steering vectors, as noted, and the microphone locations in the W-8 inlet are known very precisely. QB combines accurate peak levels with optimal resolution. The latter makes it possible to separate modal peaks in $\frac{k_{x}}{k_{0}}$, even with an axial subarray with limited length. It also gives definite values of spinning order, despite using only a half circle for the circumferential subarray. QB is also very fast, having running time that is proportional to the number of grid points, rather than a power of the number of grid points, like a deconvolution method. This is advantageous for the $m-\frac{k_{x}}{k_{0}}$ grid because this grid has many points; more than the number of cuton modes. It is convenient to use the same grid for all frequencies, with the number of grid points set to cover the range and density of the cuton modes at highest frequency of interest. A final benefit of QB is that it has high dynamic range. This is important for getting good results from the cross array, which normally has relatively high sidelobes with classical beamforming.

There are similarities between the current method and a 1984 paper by Joppa, Ref. [11]: the cross array and the idea of mapping distributions of pressure-squared in spinning order and axial wavenumber space. The main difference is that Joppa uses one subarray at a time, instead of combining them. This means that sound power calculation is not generally available, since this determination requires knowledge of both the spinning order and the radial order. Joppa also argues that a sparse array, such as the cross array, is not applicable for coherent modes, and that the duct modes are often coherent. Ref. [3] makes a case that broadband modes may not be coherent. Joppa uses an FFT instead of an advanced beamforming technique.

Lowis et al. [12] also assume that broadband modes are mutually incoherent and use an additional assumption of equal energy per mode to derive sound power estimates from a single axial array.

Haxter and Spehr [13] use wavenumber beamforming to differentiate between hydrodynamic and acoustic pressure fluctuations on a wind tunnel wall. 
In the following, duct mode theory is reviewed to establish the definitions. The connection between modal amplitude and sound power is made and the $m-\frac{k_{x}}{k_{0}}$ grid and its use are illustrated. An asymptotic directivity formula is quoted and sample results from the W-8 facility are given. Finally, additional analytical results in the companion paper [2] are noted.

\section{Duct Modes}

Consider a fan duct with uniform cross section and uniform flow in the $+x$ direction with Mach number $M \geq 0$ and sound speed $c$. The duct modes are developed using notation similar to Ref. [14]. The acoustic pressure, $p$, is assumed to obey the convected wave equation

$$
\left[\left(\frac{\partial}{\partial t}+M c \frac{\partial}{\partial x}\right)^{2}-c^{2} \nabla^{2}\right] p=0
$$

Solutions are sought in the form

$$
p(x, y, z, t)=P e^{-i \omega t} e^{i k_{x} x} E_{N}(y, z)
$$

where $P$ is a constant. The transverse eigenfunctions $E_{N}(y, z)$ obey the wall boundary conditions and

$$
\left[\left(-i \omega+i M c k_{x}\right)^{2}-c^{2}\left(-k_{x}^{2}+\nabla_{\perp}^{2}\right)\right] E_{N}=0
$$

where $\nabla_{\perp}^{2}=\nabla^{2}-\frac{\partial^{2}}{\partial x^{2}}$. Introducing the transverse eigenvalue $k_{N}$, equation [14] can be rewritten in the form

$$
\left(\nabla_{\perp}^{2}+k_{N}^{2}\right) E_{N}=0
$$

Solving Eq. (3) for $k_{x}$ gives

$$
k_{x}=\frac{-M k_{0} \pm \sqrt{\left(M k_{0}\right)^{2}-\left(1-M^{2}\right)\left(k_{N}^{2}-k_{0}^{2}\right)}}{\left(1-M^{2}\right)}
$$

where $k_{0}=\frac{\omega}{c}$.

Using the cuton ratio, $\alpha$, defined by

$$
\alpha= \pm \sqrt{1-\left(1-M^{2}\right)\left(\frac{k_{N}}{k_{0}}\right)^{2}}
$$

the dispersion relation, (4), becomes

$$
k_{x}=k_{0} \frac{\alpha-M}{1-M^{2}} .
$$

Assuming $k_{N}$ is real and $|M|<1$, Eq. (5) shows $\alpha \in[-1,1]$. As discussed in Ref. [14], for real values of $\alpha$, the sign of $\alpha$ determines the direction of energy propagation. (Reference [14] actually defines $\alpha$ using a positive square root and uses $\pm \alpha$ in Eq. 7, but a signed value of $\alpha$ is simpler for tracking the results than remembering a choice of \pm in Eq. (5) or Eq. (7)). If $\alpha>0$, then the energy of the mode propagates in the $+x$ direction, or downstream. Conversely, negative values of $\alpha$ correspond to acoustic energy propagating upstream. The functions $E_{N}(y, z)$ are normalized so that

$$
\int_{S} E_{N}^{*} E_{N^{\prime}} d y d z= \begin{cases}S, & N=N^{\prime} \\ 0, & N \neq N^{\prime}\end{cases}
$$


where $S$ is the cross section of the duct. With this normalization, the acoustic intensity of a single mode of the form of Eq. (2) is [14]

$$
I_{x}=\alpha \frac{|P|^{2}}{2 \rho_{0} c}\left(\frac{1-M^{2}}{1-\alpha M}\right)^{2}
$$

where $\rho_{0}$ is the ambient density of the air. The model assumes that all of the mechanical and flow properties are uniform in $x$ over the portion of the duct containing the phased array, which may be, for example, cylindrical, annular, rectangular or a C-shape. If necessary, the eigenfunctions could be computed from a finite difference or finite element procedure, where Eq. (4) is replaced with a finite-dimensional matrix, and the eigenvalues and eigenvectors of this matrix give $k_{N}$ and $E_{N}(y, z)$.

For an axially uniform duct, Eq. (4) implies that $k_{N}$ and $E_{N}(y, z)$ do not depend on $M, c$, or $\omega$. Adding axially uniform acoustic lining would make $P$ depend on $\mathrm{x}$. In case of a duct that varies slowly in the axial direction, such as an inlet with diffusion or a C-duct with curvature, or nonuniform acoustic lining, it may be feasible to extend the concept of a mode so that $k_{x}$ varies slowly while retaining the identity of a mode. See Ref. [15]. These features are not presently included in Beamform Interactive, so a treated or nonuniform duct would decrease the accuracy of the results.

In the case of a circular or annular duct, writing Eq. (4) in polar coordinates $(r, \theta)$, taking $E_{N}=e^{i m \theta} u(r)$, and making the change of variable $\rho=k_{N} r$, gives the Bessel equation

$$
\left[\frac{d^{2}}{d \rho^{2}}+\frac{1}{\rho} \frac{d}{d \rho}+\left(1-\frac{m^{2}}{\rho^{2}}\right)\right] u=0 .
$$

If the wall impedance is circumferentially uniform, then the solutions are

$$
E_{N}(r, \theta)=\left[A J_{m}\left(k_{N} r\right)+B Y_{m}\left(k_{N} r\right)\right] e^{i m \theta} .
$$

In the case of a hardwall, cylindrical, duct, as tested in the W-8 facility, the values are $B=0, k_{N} R=j_{m s}^{\prime}$ (the $s^{\prime}$ th root of $J_{m}(y)=0$ ), and

$$
A=\frac{2 \pi}{\left(1-\frac{m^{2}}{j_{m s}^{\prime}}\right) J_{m}^{2}\left(j_{m s}^{\prime}\right)}
$$

where $R$ is the radius of the duct (11 inches).

Note that Eq. (10) implies that $|p|^{2}$ for single mode on the wall of a hardwall cylindrical duct is independent of both $z$ and $\theta$. Let this value be denoted $|p|^{2}=b$, for reasons that will become clear. Then integrating Eq. (8) over $S$ gives the sound power of the mode as

$$
W=\alpha \frac{\pi}{2 \rho_{0} c}\left(\frac{1-M^{2}}{1-\alpha M}\right)^{2}\left(1-\frac{m^{2}}{j_{m s}^{\prime 2}}\right) R^{2} b .
$$

\section{Beamforming Approach}

An outline of the new beamforming approach can now be given. An array of wall-mounted acoustic pressure transducers is installed in a portion of an inlet or aft duct so as to measure the surface pressure at the transducer locations without disturbing the flow or the sound waves. The array has finite extend in both the axial and transverse directions, and is designed to have reasonable ability to discriminate between the modes. To economize on the number of channels, the array can be nonuniform and sparse. The most effective array designs found to date are T-shapes, combining an axial line array and a partial circumferential ring. Data are acquired with the engine or engine component simulator on condition and Welch's method is used the compute the array cross spectral matrix (CSM) for each frequency of interest. A set of steering vectors indexed by a 2D map plane is computed and a beamforming algorithm 
is used to estimate the contribution of each steering vector to the CSM. The results from the beamforming are mean square wall pressures at transducer locations, specialized to the steering vectors.

\section{A. Wavenumber grid}

In previous duct beamforming approaches, the steering vectors were values of particular duct modes, evaluated at the transducer locations. This was found to be inconvenient because a unique set of steering vectors, with a different number of cuton modes, was required for each frequency and engine speed. These approaches are also fragile because errors in the computed modes have the potential so degrade the resolution and accuracy of the final results.

In the new approach, which to date has only been implemented for hardwall, circular, ducts, a beamform map is created for a rectangular grid in the $m-\left(\frac{k_{x}}{k_{0}}\right)$ plane. The spinning order runs from $-m_{\max }$ to $m_{\max }$ with a step of 1 . In the case that the circumferential arm of the array has equal angular spacing, $\Delta \theta=\frac{2 \pi}{N_{\text {cir }}}, m_{\max }$ is usually chosen to be $\frac{N_{\text {cir }}}{2}$ to avoid ambiguities in $m$. Alternatively, a circumferential arm with nonuniform, perhaps logarithmic, spacing can be used to avoid redundancies and extend $m_{\max }$ to be sure that all cuton modes are included, even at the highest fan speeds.

The range of $\frac{k_{x}}{k_{0}}$ should be large enough to include all of propagating modes, i.e., it should extend at least from $-\frac{1}{1-M}$ (most upstream) to $\frac{1}{1+M}$ (most downstream). If the range of Mach numbers tested runs from 0 to $M_{\max }$, then the $\frac{k_{x}}{k_{0}}$ grid should include $\left[-\frac{1}{1-M_{\max }}, 1\right]$ to cover the propagating modes at any Mach number. The step in the $\frac{k_{x}}{k_{0}}$ grid should be small enough to avoid missing axial modes. This depends on the spacing of the Bessel roots through $k_{N}=$ $\frac{j_{m s}^{\prime}}{R}$ and the relation between $k_{N}$ and $k_{x}$ in Eq. (5) or Eqs. (6) and (7).

The axial transducer array should be long enough for usable resolution in $k_{x}$ and should have a sufficiently fine spacing to resolve + and - propagating waves. For equal axial spacing, Eq. (7) suggests that $\Delta x$ should be less than half of the reduced wavelength at the highest frequency, where the reduction factor is $1-M_{\max }^{2}$.

The $m-\left(\frac{k_{x}}{k_{0}}\right)$ grid is only dependent on array configuration, so it should apply for all test articles and test conditions. For each grid point, the array steering vector is computed from the axial and circumferential locations of the $N$ transducers, $\left(x_{n}, \theta_{n}\right), n=1, \ldots, N$, and $k_{0}$ :

$$
\mathbf{g}\left(m, \frac{k_{\chi}}{k_{0}}\right)=\frac{1}{\sqrt{N}}\left[\begin{array}{c}
e^{i\left(m \theta_{1}+k_{\chi} x_{1}\right)} \\
\vdots \\
e^{i\left(m \theta_{N 1}+k_{\chi} x_{N}\right)}
\end{array}\right] .
$$

Each array data CSM, C is processed using the steering vectors in the grid, $\mathbf{g}\left(m, \frac{k_{x}}{k_{0}}\right)$, and beamforming algorithm.

\section{B. Beamforming}

The simplest beamforming algorithm is

$$
b_{\left(m, \frac{k_{x}}{k_{0}}\right)}=\frac{1}{N} \mathbf{g}^{\prime} C \mathbf{g}
$$

but this has large peak width and high sidelobes. Methods such as CLEAN-SC are far superior. The newest method from OptiNav is Quantitative Beamforming, an advancement of Functional Beamforming that offers superior resolution and quantitative values along with the high dynamic range and high speed of FB. It is a form of robust adaptive beamforming [20] with a proprietary regularization technique. The assumption underlying all beamforming methods like this is that the modes are mutually incoherent. This is reasonable for broadband modes [3, 12], but unlikely for tones. Proper treatment of tone data is an inverse method instead of beamforming. The mode and grid definition above still applies. 


\section{Finding peaks}

The beamforming results are converted from the $m-\left(\frac{k_{x}}{k_{0}}\right)$ plane to the $m-\alpha$ plane by identifying peaks in each vertical $m$ colmn and assigning them to the appropriate radial orders (values of $s$ in $j_{m s}^{\prime}$ ). This identification process is more robust than computing the steering vectors directly for the modes because small errors in, say, the flow conditions, result only in displacements of the peaks in the $\frac{k_{x}}{k_{0}}$, which can be handled by using a tolerance in the peak assignment process. The use of QB is important because the peak levels are correct and the radial modes are more likely to be resolved. At this stage, Eq. (13) is used to convert the results from pressure-squared at the wall $(b)$ to sound power $(W)$.

The modal sound power maps can be processed to create several results. Summing the powers of the modes with $\alpha<0$ gives the total upstream-propagating sound power, which is the outward direction of propagation for the case of an inlet duct. The modes can be radiated to the far field using a Kirchhoff integral for an unflanged duct described by Léwy [19] giving the directivity

$$
\Phi(\theta)=\left(\frac{1}{2}+\frac{k_{0} \cos \theta}{2 k_{x}}\right) \frac{2 k_{0} R \sin \theta}{\left(k_{N} R\right)^{2}-\left(k_{0} R \sin \theta\right)^{2}} \mathrm{~J}_{m}^{\prime}\left(k_{0} R \sin \theta\right) .
$$

The far-field pressure-squared for each mode is determined from this formula and normalized to conserve the power. These results are summed incoherently to produce the final prediction of the fan noise in the far field.

The effectiveness of noise reduction technology such as over-the-rotor acoustic treatment can be estimated by comparing the in-duct sound power for the treated and untreated cases.

\section{W-8 Sample Results}

The 85 element T-shaped array of Kulite ${ }^{\circledR}$ transducers in the W-8 test is shown in Fig. 1 . The transducers in the circumferential subarray have a spacing of $4^{\circ}$, or $1 / 90$ of a circle, so the highest spinning order that that can be unambiguously resolved is $m= \pm 44$. The axial subarray has a length of 15.5 inches and a minimum axial spacing of 0.5 inches.

Sample Quantitative Beamforming results in $m-\frac{k_{x}}{k_{0}}$ plane are shown in Fig. 2. The conversion factors between the beamforming (pressure-squared) and sound power levels derived from Eq. 13 are shown in Fig. 3. The mode powers corresponding to Fig. 2 are shown in Fig. 4. The summed outgoing and incoming (reflected) sound powers level spectra are shown in Fig. 5. Evaluating the modes as functions of radius (Eq. (11)) and summing incoherently gives the radial SPL distribution shown in Fig. 6. Finally, the far field projections are shown in Fig. 7. 


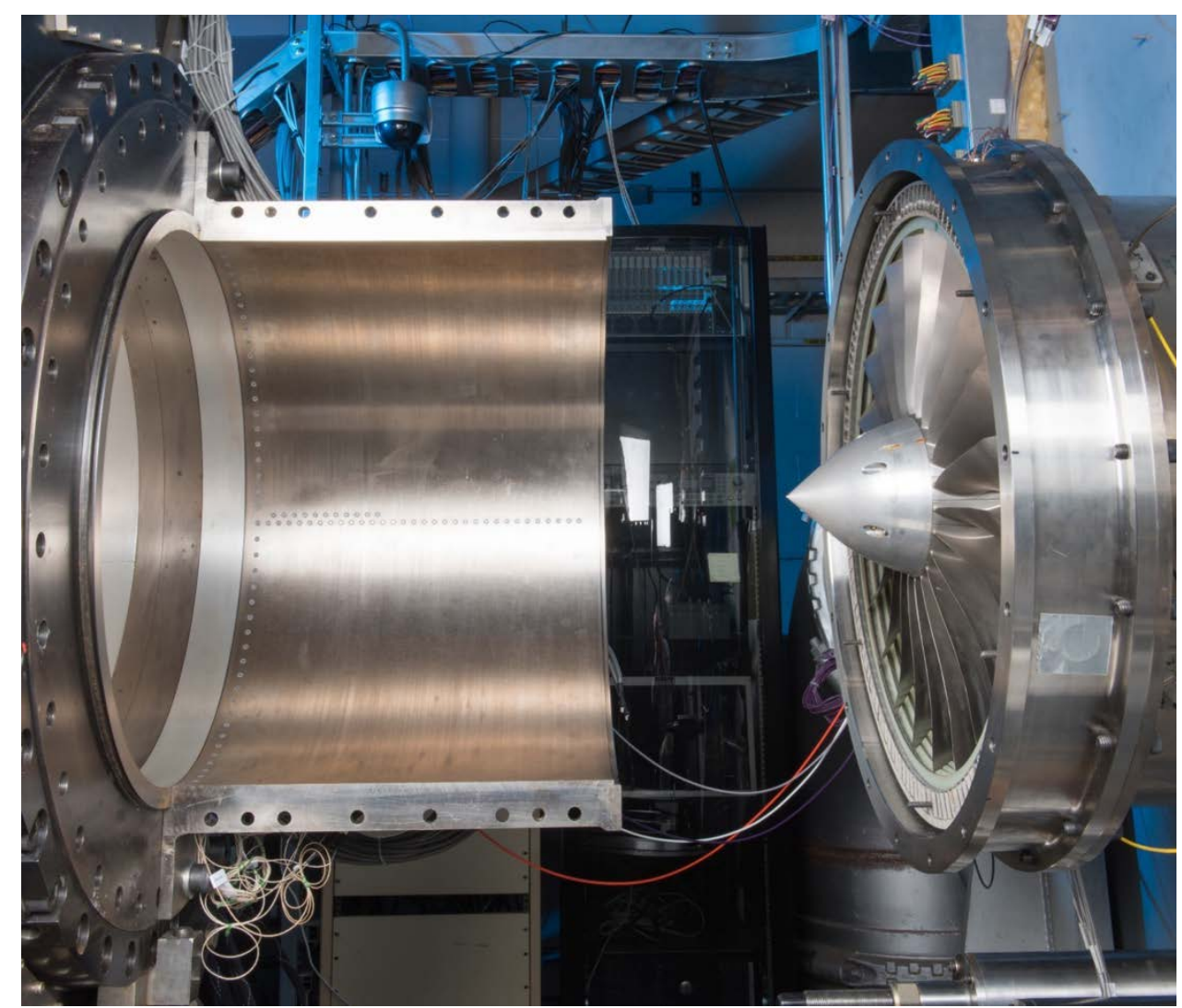

Figure 1. Transducer array for the W-8 facility.
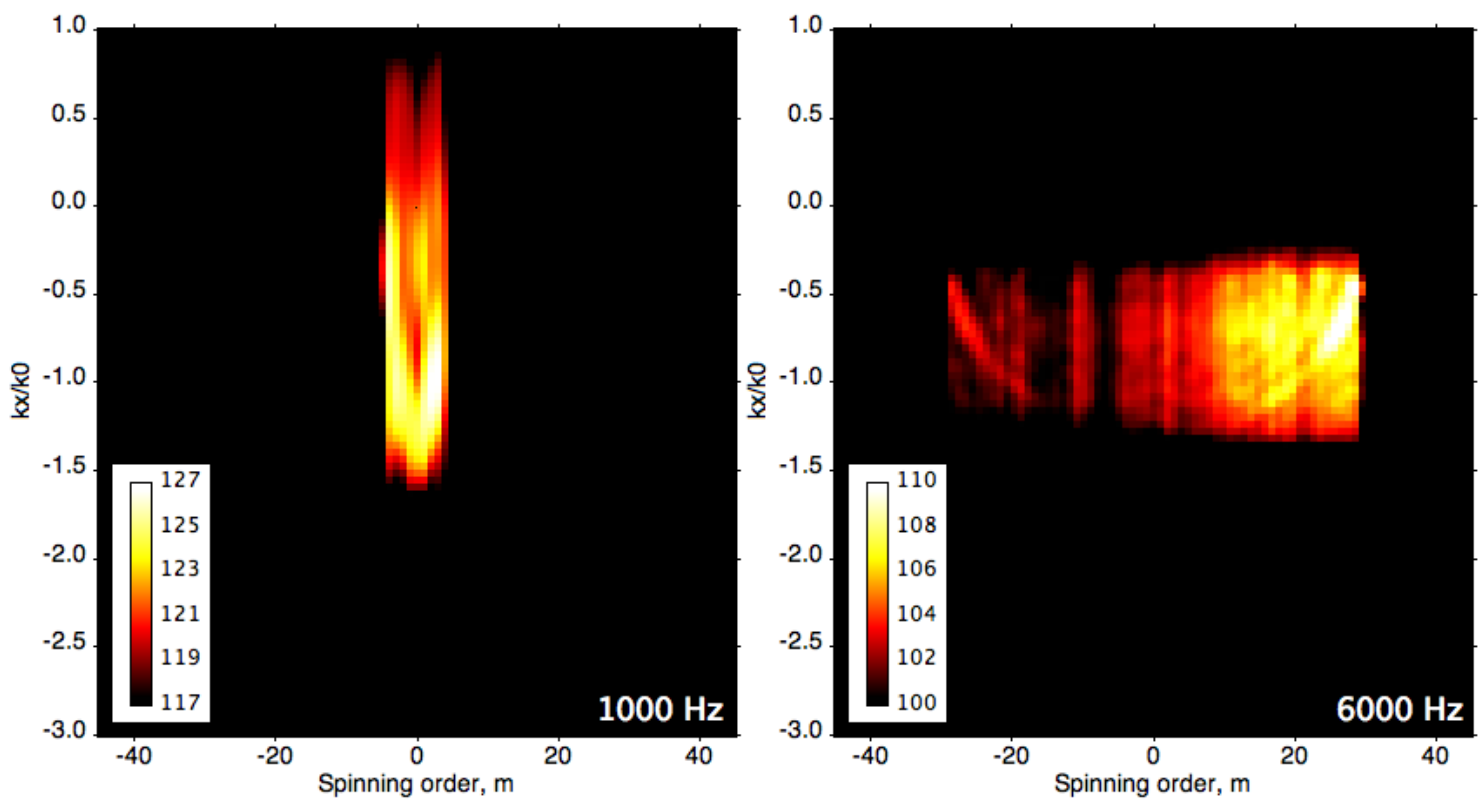

Figure 2. Quantitative beamforming for the $\mathrm{m}-\frac{k_{x}}{k_{0}}$ plane. W-8 test at $50 \%$ power. Values shown are array sound pressure level, $\mathrm{dB}$ re. $20 \mu \mathrm{PA}$. A $10 \mathrm{~dB}$ scale is range is shown to emphases the larger values. 

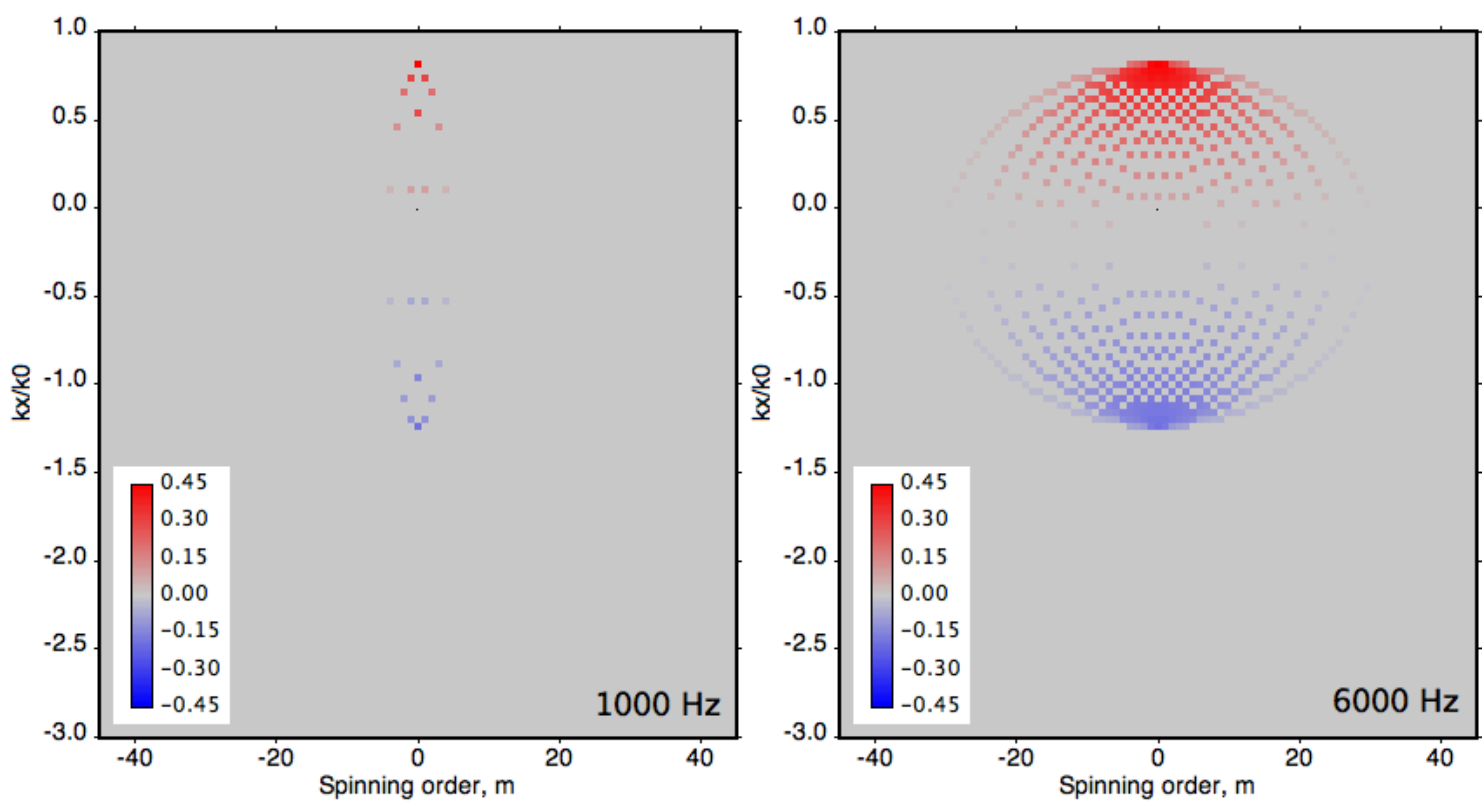

Figure 3. The conversion factor between microphone pressure-squared and mode power (Eq. 12) for the cuton modes in the W-8 test at $\mathbf{5 0 \%}$ power. Same grid as Fig. 1 . Negative power is outward propagating. Values are in $\mathbf{m W} / \mathbf{P a}^{2}$.
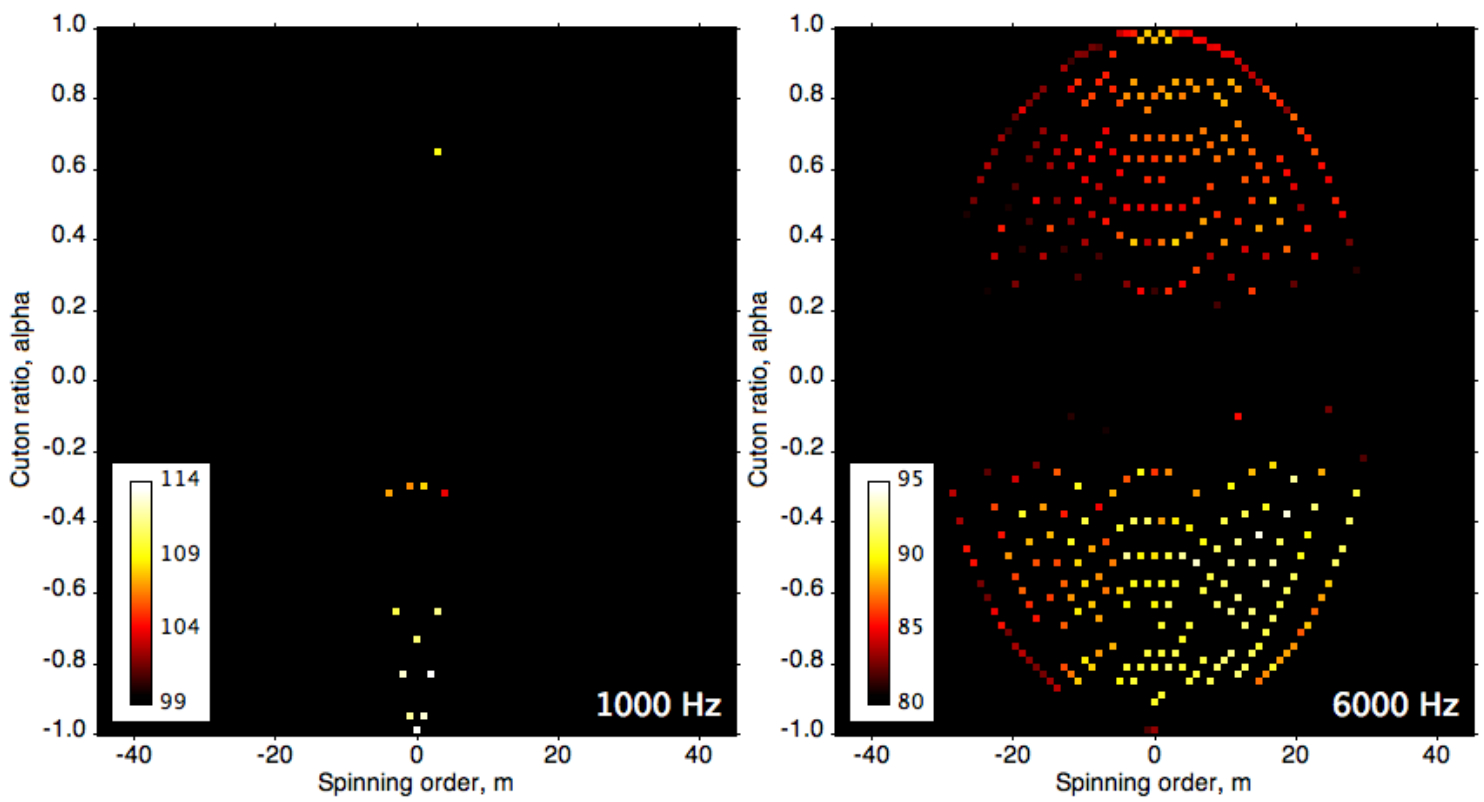

Figure 4. Mode powers derived by filtering the beamforming results in Fig. 1 to isolate peaks and applying the pressure-squared to power conversion in Fig. 2 . The vertical scale is cuton ratio; negative values represent outward propagating energy. 


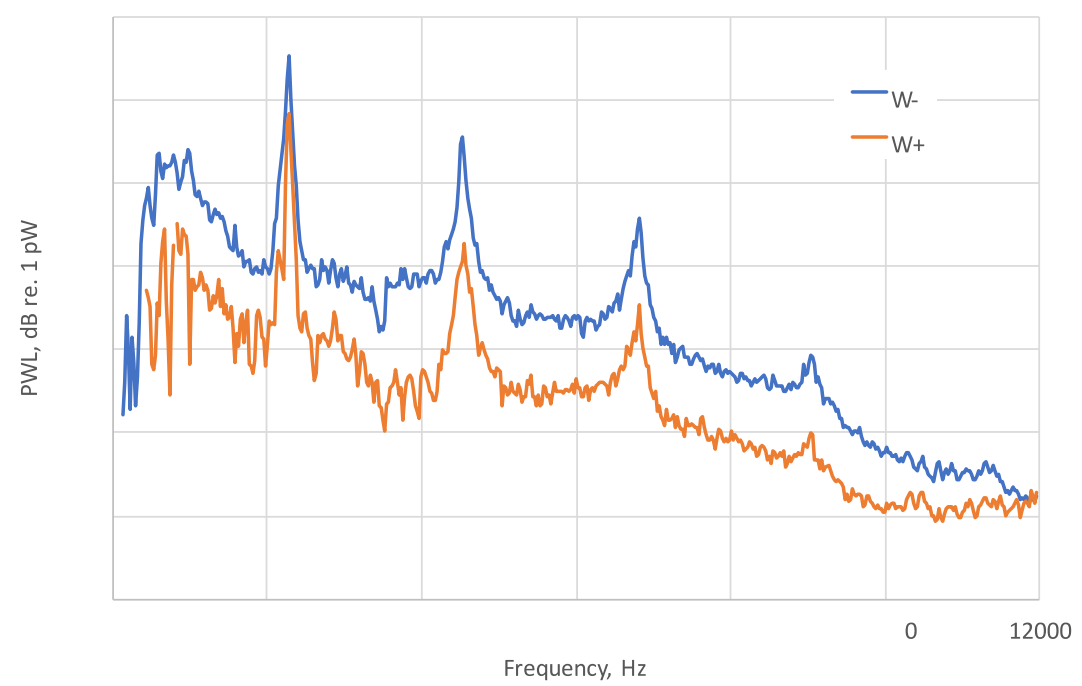

Figure 5. In duct power level for energy propagating against the flow, $\mathrm{W}-$, and with the flow, $\mathrm{W}+$.

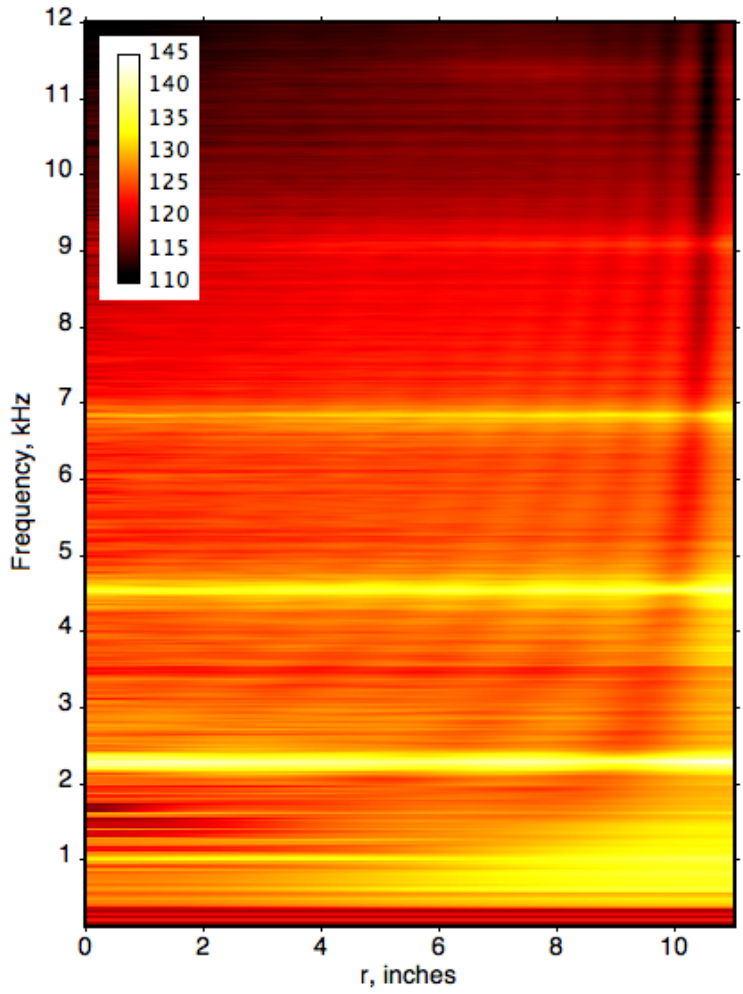

Figure 6. Radial Sound Pressure Level profile, dB re. $20 \mu$ Pa. 


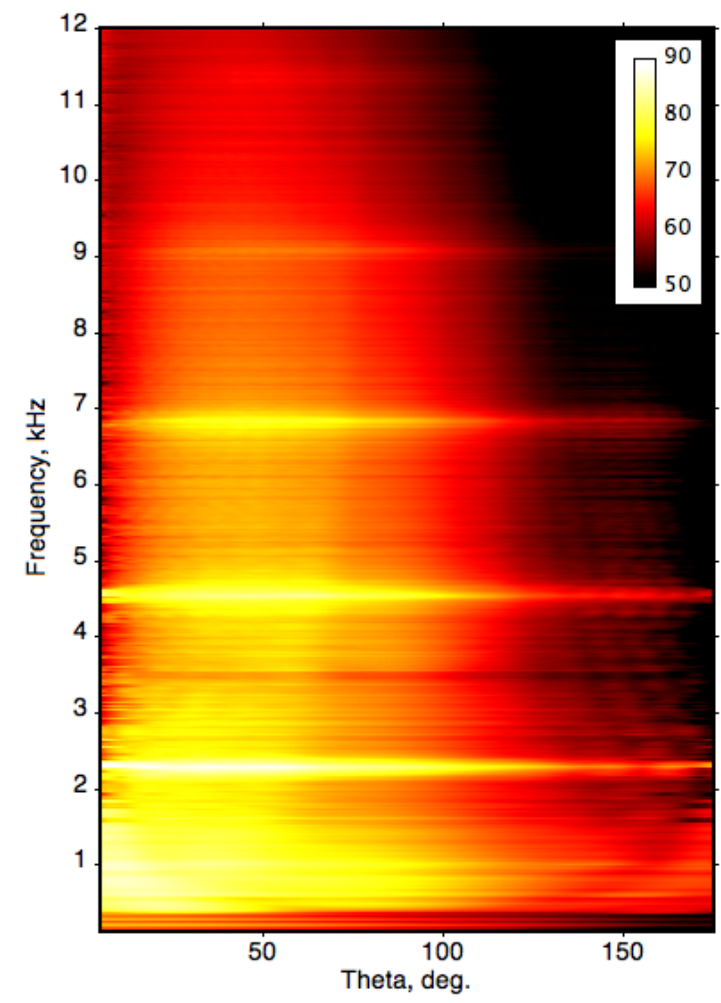

Figure 7. Lossless far field projection for $50 \%$ power, 100 foot radius., dB re. $20 \mu \mathrm{Pa}$. Inlet angle $\theta$ runs from $5^{\circ}$ through $175^{\circ}$.

\section{Conclusion}

The combination of the cross array design, the spinning order/axial wavenumber beamforming grid, and Quantitiave Beamforming gives a synergistic system that enables convenient determination of sound power and other acoustic quantities in a uniform, hardwall flow duct such as NASA's W-8 facility. Growth paths are available to extend the method to important cases of acoustic treatment, nonuniform ducts, and tones.

\section{Acknowledgments}

This work was supported by the Advanced Air Transport Technology Project of NASA's Fundamental Aeronautics Program. R.P. Dougherty would like to thank Dan Sutliff, Bruce Walker, Don Weir, Jeff Mendoza, Ann Heyward, and Gary Podboy for support over a period of years leading to this capability.

\section{References}

[1] Van Zante, D.E, Podboy, G.G., Miller, C.J., and Throp, S.A., "Testing and performance verification of a high bypass ratio turbofan rotor in an internal flow component test facility”, NASA/TM-2009-215661, 2007.

[2] Bozak, R.F. and R.P. Dougherty, "Noise Reduction from Acoustic Casing Treatments Installed Over a Subscale High Bypass Ratio Turbofan Rotor”, AIAA Aeroacoustics Conference, 2018.

[3] Dougherty, R.P., “Mutual Incoherence of Broadband Duct Acoustic Modes”, AIAA Paper-3032, May-June 2016

[4] Dougherty, R.P. and Mendoza, J.M. "Nacelle in-duct Beamforming Using Modal Steering Vectors”, AIAA Paper 20082812, May 2008.

[5] Dougherty, R.P., Walker, B.E., and Sutliff , D.L, "Locating and Quantifying Broadband Fan Sources using In-Duct Microphones”, AIAA Paper 2010-3736, 2010.

[6] Marotta "Validation of Beamforming Analysis Methodology with Synthesized Acoustic Time History Data: Sub-Scale Fan Rig System”, AIAA Paper 2014-3068, June 2014.

[7] Tapken, U., Gutsche, D., and Enghardt, L., "Radial mode analysis of broadband noise in flow ducts using a combined axial and azimuthal sensor array”, AIAA Paper 2014-3318, June 2014.

[8] Sijtsma, P., "CLEAN based on spatial source coherence," Int. J. Aeroacoustics, Vol. 6, No. 4, 2009, pp 357-374.

[9] Brooks, T.F. and Humphreys, W.M. Jr., "A Deconvolution Approach for the Mapping of Acoustic Sources (DAMAS) determined from phased microphone arrays,” AIAA Paper 2004-2954, 2004. 
[10] Dougherty, R.P., "Functional Beamforming for Aeroacoustic Source Distributions", AIAA Paper 2014-3066, 2014.

[11] Joppa, P.D., “An Acoustic Mode Measurement Technique”, AIAA Paper 84-2337, 1984.

[12] Lowis, C.R., P.F. Joseph and A.J. Kempton, "Estimation of the far-field directivity of broadband aeroengine fan noise using an in-duct axial microphone array”, J. Sound. Vib. 2010, Vol. 329, pp. 3940-3957.

[13] Haxter, S. and Spehr, C., "Infinite beamforming: wavenumber decomposition of surface pressure fluctuations", Berlin Beamforming Conference, BeBeC 2014-04, 2014.

[14] Morfey, C.L. "Sound transmission and generation in ducts with flow”, J. Sound. Vib. 1971, Vol. 14, No. 1, pp. 37-55.

[15] Oppeneer, M., Lazeroms, W.M.J., Riensra, S.W., Matheij, R.M.M., and Sijstma, P., "Acoustic modes in a duct with slowly varying impedance sna non-uniform mean flow and temperature,” AIAA Paper 2011-2871, June 2011.

[16] Marotta, T.R., L.S. Lieber, and R.P. Dougherty "Validation of Beamforming Analysis Methodology with Synthesized Acoustic Time History Data: Sub-Scale Fan Rig System, AIAA Paper 2014-3068, 2014.

[17] Dougherty, R.P., B.E. Walker and D.L. Sutliff, "Locating and quantifying broadband fan sources using in-duct microphones”, AIAA Paper 2010-3736, 2010.

[18] Dougherty, R.P., “Mutual Incoherence of Broadband Duct Acoustic Modes”, AIAA Paper 2016-3032, Lyon, France, 2016.

[19] Léwy, S., "Inverse method predicting spinning modes radiated by a ducted fan from free-field measurements", J. Acoust. Soc. Am. 20305, Vol. 117, No. 2, pp. 744-750.

[20] Li, J. and Stoica, P., Robust Adaptive Beamforming, Wiley, 2006. 